Voix et Images

voixetimages

\title{
Précieux et préciosité chez Bessette : demi-mesure et démesure
}

\section{Françoise Iqual}

Volume 1, numéro 3, avril 1976

Gérard Bessette

URI : https://id.erudit.org/iderudit/200036ar

DOI : https://doi.org/10.7202/200036ar

Aller au sommaire du numéro

\section{Éditeur(s)}

Les Presses de l'Université du Québec

\section{ISSN}

0318-9201 (imprimé)

1705-933X (numérique)

Découvrir la revue

Citer cet article

Iqbal, F. (1976). Précieux et préciosité chez Bessette : demi-mesure et démesure. Voix et Images, 1(3), 338-364. https://doi.org/10.7202/200036ar d'utilisation que vous pouvez consulter en ligne.

https://apropos.erudit.org/fr/usagers/politique-dutilisation/ 


\section{Précieux et préciosité chez Bessette: demi-mesure et démesure}

La préciosité se révèle être dans l'œuvre romanesque de Gérard Bessette une constante. De prime abord, cette préciosité a en commun avec celle du XVIIe siècle français une continuelle recherche au niveau de l'expression. Cette recherche qui ressortit à la "fixation linguistique et stylistique ", jadis évoquée par Bessette lui-même ', se traduit par un retour d'invariants dans chacun des romans. Au nombre de ceux-ci figure le recours au terme et à l'expression qui tendent à être recherchés, didactiques ou érudits; viennent ensuite l'insertion d'argot et de mots familiers propres surtout à la France, la référence à des noms d'écrivains et enfin le rappel d'œuvres littéraires ainsi que la citation occasionnelle d'une ligne célèbre.

A ces particularités, que le lecteur ne peut manquer de déceler, se joint un goût des «embellissements", lequel, pour être sans cesse présent, ne se manifeste pourtant pas avec la même transparence. Ces «embellissements" s'identifient aux détails, digressions et explications intercalés dans un récit, non dans le but de contribuer au développement de l'intrigue, mais pour le plaisir esthétique d'utiliser un vocabulaire pertinent ou de jouer avec les mots. Ainsi la Bagarre ${ }^{2}$ multiplie les descriptions dont plusieurs trahissent une appartenance à l'exercice scolaire, telles ces évocations de lieux: la Bougrine, l'Anchor, la salle de quilles, la salle d'attente d'Hochelaga, l'extérieur et l'intérieur des maisons... Le Libraire pour sa part ne manquera jamais, dans son combat contre une langue étriquée, de se payer la jouissance d'une précision des termes analogue à celle-ci :

Il a le teint naturellement cireux. Mais ce matin-là, il exagérait: verdâtre ou cadavérique le décriraient mieux. Des rides profondes, creusées par la fatigue, lui ravinaient le front et les méplats. Une barbe pisseuse lui végétait sur le menton tandis que des fibrilles rouges lui zébraient le blanc des yeux ${ }^{3}$.

Dans les Pédagogues ${ }^{4}$, c'est la minutieuse description des gestes et évolutions de la danseuse siamoise ( $p .70-71$ ), c'est la soirée de la ministresse (p. 129-174) qui toutes deux favorisent des hors-d'œuvre verbaux que ne démentirait pas un Cyril Arbour. Avec l'Incubation, non seulement les séries synonymiques fourmillent mais la pratique du «divertissement littéraire» et 
de l'ornatus y est monnaie courante. On y croise fréquemment une telle arabesque :

il parlait sans doute des chevaux à moins que ce ne fût des femmes, c'était d'ailleurs sans importance attendu que personne ne l'écoutait sauf peut-être une petite femme [...] à la chevelure de saule pleureur (comme elle disait: à la weeping-willow) laquelle (chevelure) cotonneusement érigée au-dessus du sinciput lui tombait ensuite en mèches branchues sur les épaules le dos la poitrine jusqu'au niveau (théorique) des seins [...] elle poussait cette femme-saule, chaque fois que Ripcord faisait mine de poser la main sur son arcature capillaire, des cris striduleux... 5 .

ou semblable ramification, prodige de virtuosité intellectuelle et d'ironie:

dans ce sanctum sanctorum philologique où la bi-hebdomadaire femme de ménage calabrienne (qui parlait ach un italien indescriptible) ne pénétrait qu'avec crainte et tremblement pour effleurer du bout de son plumeau (c'était touchant et comique Weingerter s'en tenait parfois métaphoriquement bien sûr métaphoriquement les côtes) comme s'il se fût agi d'explosifs Menschenkinger de $H$ bombs, les longues feuilles quadrillées (en prévision d'éventuels graphiques) couvertes de pattes de mouche, alors que pourtant nicht wahr la dérivation onomatopéique n'avait jamais tué personne jamais blessé quiconque sinon métaphoriquement ${ }^{6}$.

Le Cycle enfin se plaît à l'accumulation de mots et au maniérisme formel à la recherche de l'alambiqué, aussi un morceau de bravoure comme celui qui suit s'y répétera assidûment:

autour de toi maintenant ex-pater familias (exploité jusque dans la mort) autour de ces cierges absurdes et de ce crucifix contorsionné s'agenouillent et prient des sous-hommes des sous-femmes domestiqués conditionnés abrutis par l'étouffant système claustrophobique [...] grands oncles campagnards aux gestes de robot à la peau tannée comme du cuir parentèle loufoque irrécupérables crétins injectés au hasard dans le creuset ventral puis éjectés spasmodiquement (boules de chair hurlantes et glaireuses) d'une chair sanglante et meurtrie (vagissements dérisoires de l'homoncule bleu faisant vibrer pour la première fois l'irrespirable atmosphère terrestre $[. . .]^{7}$ )

De tels invariants et embellissements se rattachent en raison de leur filiation avec la rhétorique à l'aspect formel de la préciosité. Or, chez Bessette, cette dernière est aussi fond. Elle semble du reste, dans les trois premiers romans, se fonder davantage sur le fond pour ensuite se ranger principalement sous la bannière de la forme dans les deux derniers romans. Qu'il s'agisse cependant d'une manière ou de l'autre, le fait demeure que la préciosité se manifeste, au niveau du fond, par l'entremise d'un personnage dont l'attitude correspond à celle du précieux. Ce personnage se retrouve dans chacun des romans et se nomme tour à tour Augustin, Jodoin, Lambert, Weingerter et Julien. Même si le nom, l'âge et l'occupation varient d'un récit à l'autre, le personnage se découvre à l'analyse fondamentalement identique. Nous chercherons donc à la définir en étudiant d'abord individuellement chacun des précieux de sorte que nous pourrons ensuite consigner les lignes de force qui se dégagent de leur rapprochement. Comme un rapport étroit se dessine entre le précieux et le langage, les 
considérations sur le fond nous ramèneront en dernier lieu à des réflexions sur la forme dont nous envisagerons l'évolution dans l'œuvre romanesque de Bessette.

\section{LA BAGARRE: AUGUSTIN SILLERY}

Dès sa première apparition dans le roman, cet étudiant en lettres fait une entrée en scène théâtrale sur laquelle se modèleront maintes de ses entrées subséquentes: bras étendus, voix aiguë, il s'exprime en "doctes palabres ". Autant que la pose du personnage, le contexte importe ici. Augustin se présente au café en compagnie du "petit ami " Langevin et interrompt, par ses propos oiseux, la discussion d'un projet de thèse sur les Canadiens français entamée par deux confrères d'université, Lebeuf et Weston. Voilà un point de départ qui rassemble la question du sexe, du langage, de l'identité canadienne-française et qui confronte les personnages antagonistes de Lebeuf et Sillery. Le lecteur se trouve donc, à son insu, dès la rencontre initiale avec Augustin qui se situe au début de la narration, mis en présence des éléments qui à la fois feront se dérouler le récit et permettront de comprendre le fonctionnement des rouages de la préciosité. II y a en effet dans cette scène un trait frappant, le jeu de mutuelle exclusion entre Jules et Augustin. Ce jeu ne connaîtra de cesse par la suite si bien que l'on en déduit que l'un incarne une thèse dont l'autre est l'antithèse. Ainsi, au niveau de l'expression, le premier est tourné vers une langue simple et directe et le second séduit par la tentation de l'éblouissement et du raffinement. Une des règles d'un tel jeu sera l'exploitation de la vulnérabilité de l'autre, d'où la parole d'Augustin raille les aspirations littéraires de Jules tandis que le silence de ce dernier crie au dandy son homosexualité. On est.alors amené à se demander quelle est cette parole et quelle est l'origine de ce silence?

Dès qu'elle s'adresse à Jules et à son double Weston ${ }^{8}$, auditeurs initiés parce qu'étudiants en littérature, la parole d'Augustin se doit d'étinceler: c'est véritablement le “bel esprit», marqué en public d'une “déformation professionnelle". Le raffinement des mots cherche chez lui à s'allier celui de l'esprit, ce faisant les évocations littéraires et linguistiques prolifèrent depuis l'allusion à "la dive bouteille", en passant par la réminiscence d'une ligne d'Athalie ( $p$. 10), jusqu'à l'appel au "dérèglement rimbaldien" (p. 11). Non content de semer son discours inaugural de fleurs littéraires, Augustin le sème encore de considérations psychologiques relatives à l'CEdipe, à la libération du moi et à l'inauthenticité. II y a du. reste une insistance littérale sur l'inauthenticité car à deux reprises reparaît explicitement la référence implicite à Sartre que cette notion suppose ${ }^{9}$. Pareille insistance et l'importance des passages où s'insère cette référence en insinuent la fonction de connotation.

Puisque l'CEdipe vient d'être invoqué, soulignons qu'Augustin est enfant adulé de sa mère. En effet, non seulement celle-ci supporte en silence et en souffrance à demi dissimulée ses absences et écarts de conduite, mais encore elle s'avoue sa complice dans le conflit qui l'oppose à 
son père. Jusqu'où n'irait pas la solidarité d'une mère et d'un fils intellectuels face à un mari, homme d'affaires terre à terre occupé à "additionner des colonnes de chiffres, acheter, vendre, surveiller le marché " ( $p .33)$, qui a le tort de reprocher à son rejeton littéraire de ne savoir que peser "des pattes de mouche dans des balances de fils d'araignée" (p. 33)!

Par suite sans doute, entre autres raisons, de la situation familiale, Augustin est homosexuel. Cette "condition" s'identifie dans son esprit à une tare et devient dès lors source d'un complexe d'infériorité:

II ne pensait pas seulement à Langevin. Langevin' n'était qu'une conséquence. II pensait à sa "condition": à lui-même, en somme, à son essence, à sa vie... "Pourquoi moi ? Pourquoi pas les autres ?" Puis il essayait de se résigner [...] Rien à faire? - pas si sûr. C'était une particularité morale, pas physique... Éternelle question du libre arbitre [...] Augustin se rappela Guerre et Paix, l'argumentation de Tolstoï : impression de liberté à l'intérieur de la conscience; rigide enchaînement des effets et des causes dans le monde extérieur. "Insoluble, mais corrosif." (p. 75-76).

Infériorité qu'il cherche à masquer et qui se double de culpabilité envers lui-même, puisqu'il pressent sa part de responsabilité, et à l'égard de sa mère dont il abuse en perpétuant un comportement d'enfant faible et choyé. À leur tour, infériorité et culpabilité se conjuguent à un sentiment de solitude ( $p .35$ ), d'où, chez Augustin, une perpétuelle inquiétude qui se trahit dans des signes physiques: mains moites ou brûlantes, jambes molles, transpiration du corps... Cette inquiétude, intimement liée à la crainte du jugement d'autrui et de son mépris, le porte paradoxalement vers cet autrui, l'obstacle à vaincre ou à conquérir. Qui est cet autrui ? Dans la Bagarre, autrui a pour nom principal Jules Lebeuf ${ }^{10}$, "colosse" à double dimension, collective et individuelle. Considéré sous l'angle de la collectivité, il devient un symbole de la problématique du Canadien français à la recherche de son identité. Envisagé du point de vue de l'individu, c'est l'être partagé entre l'idéal humanitaire de la préparation d'un avenir plus juste pour le travailleur et le vieux rêve de devenir écrivain, l'idéal s'avérant sans cesse déçu par l'application et le rêve impossible à réaliser puisque Jules est incapable de faire coïncider le temps de la vie avec celui du livre à écrire:

Que devenaient dans tout ça ses rêves littéraires, son vague désir d'aider ses compatriotes, de réveiller l'âme montréalaise?... Bien qu'il eût du temps libre durant la journée, Jules n'avait pas retouché à son "roman". II prenait moins de notes qu'auparavant. "Faut pourtant que je m'y remette un de ces jours..." Souvent le colosse regrettait sa vie d'étudiant, cette vie d'insouciance et d'irresponsabilité, les longues séances dans les cafés où l'on buvait $s e c$ et discutait ferme... Cependant, ce n'était pas ça, la vraie vie, non plus. "Faut que je me remette à écrire un de ces jours... " (p. 231).

Le dualisme de Jules est nourri, comme on peut le constater tout au long du roman, par l'histoire canadienne et québécoise. Il est du reste frappant que l'idéologie dont il est porteur demeure fondamentalement conservatrice malgré certaines allures révolutionnaires et son caractère de transition. Aussi, ce dualisme renvoie en dernière analyse aux notions antithé- 
tiques du bien et du mal, de la lumière et de l'ombre, de l'individu et de la société et ces notions représentent les valeurs "d'un petit groupe francophone perdu dans un coin de l'Amérique du Nord [...] des paysans pour la plupart, sans instruction [...] [qui] avaient dû aller au plus pressé: vivre, cultiver la terre, défendre leurs traditions, leur langue" (p. 189-190). Pour Augustin, cet autrui-Jules, préoccupé de survivre plutôt que de vivre, s'assimile au mal inhérent au milieu «désorganisé, inconscient, sans cohésion» (p. 58) dont il est issu et à la non-parole que concrétise son impuissance à écrire. Lebeuf est fond sans forme comme le révèlent sa tentative scripturaire (p. 94-98) et son échec en philologie. II est, ce faisant, partie d'un univers figé appelé à la dissolution comme l'illustre l'image que ses derniers mots font surgir à la fin du roman: "C'est idiot de rester ainsi immobile sous la pluie. " (p. 231)

Projeté dans le mal et la non-parole, Augustin est parallèlement incapable de les accepter ou de les refuser. II restera coincé entre l'adhésion aux idées et mœurs des "bien-pensants" et l'appel de sa sexualité, d'où, au niveau psychologique, sa "mauvaise conscience". Cette dernière le rend coupable de "mauvaise foi" en lui faisant renoncer à l'authenticité car, ne cherchant pas à s'engager, il s'en remet à la morale établie, qu'elle revête ou non l'apparence d'une fuite rimbaldique "dans les déserts d'Afrique " (p. 228) : authentique produit ambivalent d'une société aliénée.

Au niveau du langage, l'être coincé n'aborde pas autrui par voie directe mais par le biais de la préciosité. Ce moyen terme revêt ici la forme d'une prolixité verbale, proche parente, dans son goût de l'ostentation et dans l'attachement aux citations et références littéraires, de la logomachie. À la réflexion, le beau langage d'Augustin et son enivrement de mots font figure de refuge contre une réalité hostile et opprimante". " personnifie une tentative d'affranchissement et de dépassement qui échouera parce qu'incomplète comme est incomplète son argumentation en faveur de la philologie pour les Canadiens français ${ }^{12}$. II devient, dans cette optique, intéressant de constater qu'Augustin ne réussit pas à créer la présence et à réaliser la communion auxquelles pourtant se rattachent les figures de rhétorique caractéristiques de son discours, répétition, amplification ${ }^{13}$, allusion et citation littéraires, apostrophe ${ }^{14}$ :

Parmi les figures ayant pour effet d'augmenter le sentiment de présence, les plus simples se rattachent à la répétition [...] L'effet de présence s'obtient, bien plus que par une répétition litterale, par un autre procédé qui est l'amplification 15 .

Les figures de communion sont celles où, au moyen de procédés littéraires, l'on s'efforce de créer ou de confirmer la communion avec l'auditoire. Souvent cette communion est obtenue grâce à des références à une culture, une tradition, un passé communs. [Parmi ces figures, les auteurs citent l'allusion, la citation et l'apostrophe ${ }^{16 .}$.

II semble, à la lueur de ces constatations, que la "voix" d'Augustin essaie de substituer à l'absence et au silence québécois une présence et une parole. Si l'effort d'union et d'expression se résout en échec, c'est que sa parole, affirmation matérielle du langage de par son éclat même, ne s'identifie 
pas à la réalité québécoise. Elle nie cette dernière plutôt que de l'intégrer, ainsi, bien qu'âme-sœur de Nelligan, Augustin ne cite pas le poète canadien mais s'appuie sur des écrivains français, laissant à l'Américain Weston l'évocation de nos auteurs. Le récit corroborera donc à plusieurs reprises que sa parole sonne faux: "Sillery reprit d'une voix de fausset, d'une voix tragique ( $p .10,63)$, interrompit d'une voix aiguë, voix étrangement perçante (p. 10,56), répondit de sa voix flûtée (p. 62-63), la voix d'Augustin paraissait encore plus artificielle (p. 212)... " C'est un fait que la langue d'Augustin se montre, comme sa voix, au fur et à mesure que progresse la narration, de plus en plus superficielle. Sa conversation loufoque s'éloigne désormais de la littérature pour se rapprocher de ce journalisme dont il dit que "[l]e nec plus ultra [...] consiste à pondre des articles ex nihilo" (p. 215). Le jeu avec les mots paraît l'emporter sur le jeu avec les idées ${ }^{17}$. Devant cette démesure qui traduit une demi-mesure, on recoupe au niveau linguistique la "facticité existentielle" discernée au niveau psychólogique. Augustin ne transcende pas l'expérience nocturne du naufrage. Substituant, au lieu de les marier, la jouissance verbale/ "petite masturbation intellectuelle» (p. 20) à la jouissance sexuelle, il demeure à la gestation en demeurant au "C'était pendant l'horreur d'une profonde nuit " qui revient si fréquemment sur ses lèvres.

Augustin a laissé à autrui le soin de créer son moi, autrui en qui vivent les forces de la tradition, les négations de la survivance, les tiraillements de l'ambiguïté, aussi le masque du beau langage et du bel esprit qu'il avait emprunté n'a pas collé à sa peau mais est peu à peu tombé. Masque qui courtisait la démesure ${ }^{18}$ au point de départ, il a en cours de route bifurqué vers la demi-mesure puisqu'il n'a endossé ni la réalité, ni la scène d'où, chez celui qui l'arborait, disjonction et errance. L'acteur eût-il fait vraiment sienne cettè démesure, il aurait réussi à surmonter son dualisme. Son ivresse n'aurait alors pu être assimilée à «une ivresse standard, accompagnée de vomissements plus ou moins prolongés" (p. 63) - tirades-jeux verbaux, interrogations existentielles point approfondies - car elle se serait transformée en ivresse dionysiaque. "Dive bouteille" au "dive contenu", l'ivresse aurait été ce qu'est le dieu dont elle tire son nom: "Dionysos porte le nom hautement significatif de 'libérateur' 19 " Lorsque I'homme, en effet, se laisse pénétrer par le dieu, tout se passe comme lorsque celui-ci fait son entrée dans le monde:

Le monde familier [...] n'est plus! Le déchaînement suscité par l'arrivée de Dionysos l'a balayé. Tout est métamorphosé [...]. C'est le monde originel qui est venu au premier plan: les profondeurs de l'être se sont ouvertes, les formes premières de tout ce qui est créateur et destructeur ont surgi [...]. Elles ont fait voler en éclats l'image paisible d'un monde bien ordonné et sans surprise [...]. Tout ce qui était fermé s'ouvre. L'étranger et l'hostile s'ajointent en un accord miraculeux...20.

En devenant célébrant du culte de Dionysos, nommé parfois "l'efféminée, parfois "androgyne ${ }^{21}$ ", Augustin aurait réalisé la conciliation des oppositions : " tout ce qui vit [...] surmonte la séparation entre lui-même et son opposé 22 ». 
Seule la démesure pouvait provoquer sa participation à la bagarre contre les forces traditionnelles et assurer la réconciliation du corps et de l'esprit, lesquels demeurent dans le roman irréductible antinomie ${ }^{23}$.

\section{LE LIBRAIRE: HERVÉ JODOIN}

Malgré ses allures d'anti-précieux, Jodoin n'en fraie pas moins sans cesse avec la préciosité, mais cette fois une préciosité retournée. C'est avec tant d'application qu'il feint d'inverser les valeurs établies, de mépriser bourgeois et femmes, de se séparer de son prochain que pareil comportement n'est pas dépourvu d'affectation. II est en effet probant que ce libraire est passé maître en simulation et dissimulation et que sa maîtrise lui assure un masque en même temps qu'elle lui procure une jouissance intellectuelle.

Que Jodoin soit un acteur, les exemples abondent. Le lecteur n'a qu'à se référer à «l'interview d'opéra-comique» (p. 25) avec Martin Nault, à la conduite adoptée avec les trois vieilles filles, collègues de travail, à ses "recettes " de tranquillité en librairie, au rituel de la taverne, à la scène burlesque avec le curé, aux échanges avec Rose, à la conversation avec le chef de gare ou à la duperie finale de Chicoine. Ce talent versatile s'avoue à l'observation un cabotin au sens moderne du mot. Il est et joue tout à la fois le personnage qu'autrui désire retrouver et que lui-même aspire à devenir ou à projeter: "calme, stoïque, taciturne [...] sage» (p. 164) comme le père Manseau. On perçoit, au fil de ses notations, que l'être et le paraître furent jadis en conflit chez lui et le demeurent. Pourquoi et comment en est-il ainsi sont alors les questions qui surgissent naturellement à l'esprit. Dans la tentative d'y apporter une réponse, on s'attardera d'abord à ce que veut être Jodoin: arithmomane et humoriste.

Jodoin se présente comme un fervent de l'arithmomanie, une arithmomanie qui l'entraîne dans une certaine direction, celle de s'imposer des limites. Dans le domaine spatial, cette exigence des limites instaurera une réduction de l'espace qui va de pair avec une haine du mouvement. Ainsi, la chambre louée à Saint-Joachin "n'est pas grande" (p. 11) et a l'avantage d'être située non loin de la librairie et de la taverne, seuls endroits que hante Jodoin. À la librairie, ce dernier n'aspire qu'à s'installer «sur un tabouret derrière le comptoir, les mains au menton, la visière rabaissée sur le nez, et [ne rien faire] " ( $p .38$ ). A la taverne, l'endroit privilégié est « un coin, contre une bouche d'air chaud, près des latrines" (p. 13) car "c'est l'endroit le plus chaud et celui qui [lui] demande le moins de déplacement quand [il] doit[t] aller [se] soulager» (p. 13). À l'espace mesuré et clos correspond sur le plan temporel un désir d'abolir le temps, littéralement ade tuer le temps" (p. 11). II s'y appliquera au moyen d'une vie réglée par une routine étroite et rigoureuse. La continuelle répétition qui en dérive, excluant l'imprévu pour ne laisser place qu'au semblable, assure une uniformité analogue à l'immobilité temporelle: “Je ne fais rien. J'attends l'heure de la fermeture, cinq heures et demie. Il est étonnant comme le temps passe 
vite quand on ne fait rien. Pourvu qu'on ne soit pas libre. Je veux dire: pourvu qu'un 'devoir' vous force à rester en place... ${ }^{24}$ (p. 38-39)

Cependant, lorsque Jodoin entreprend d'écrire son journal "pour tuer le temps le dimanche quand les tavernes sont fermées" (p. 14), voici que l'expression "tuer le temps" devient source de paradoxe. Alors qu'elle tendait, ci-haut dans le champ de l'agir, vers le vide, c'est à la poursuite du plein qu'elle s'emploie dans le champ de l'écriture puisque le but visé est de remplir un temps mort. Si on considère par la suite la matière du remplissage - le journal est un nouvel Essai sur les mœurs et l'esprit, cette fois de la nation québécoise - l'expression dévoile une valence autre: elle se fait appel à l'ensevelissement d'un temps historique, historicité qui s'identifie désormais à la nuit d'une collectivité puisque, coulée dans le moule de l'intolérance, elle est devenue pétrification. Le projet de Jodoin équivaut ainsi au célèbre "Écrasons l'infâme" et sa contestation des valeurs traditionnelles du système, incarnées dans les personnages de Nault, de Chicoine, des demoiselles Galarneau, Placide, Morin, du curé et de Rose, en démontrera méticuleusement les insipidités.

L'entreprise de dévoilement, édifiée sur le paradoxe de «tuer le temps", devient à son tour paradoxale car source du conflit ci-dessus mentionné entre l'être et le paraître de Jodoin. Ce paraître souscrit en effet à l'idéal d'insensibilité, mais cette insensibilité recouvre une profonde sensibilité. Certains mouvements vers autrui l'attestent, à savoir la réponse à la serveuse qui lui a donné la carte de Saint-Joachin, la vente de l'Essai sur les mœurs au collégien "dévoreur" de livres comme lui le fut jadis, son intimité avec Rose et la sortie au Palace, les salutations-inclinaisons de tête et la poignée de mains finale au père Manseau. Certaines propensions à la loquacité en témoignent également, telle la «harangue sur la liberté " (p. 69) lors de l'introduction au capharnaüm ${ }^{25}$, tel le «tour de force» (p. 83) verbal déployé à l'occasion de la visite du curé ou la défense du "lecteur sérieux" (p. 117-118). Du reste, dans une perspective d'ensemble, la pénétration et la perspicacité des observations d'un Jodoin écrivain dénotent la profondeur de sa sensibilité. Ainsi, un être existe qu'un paraître d'impassibilité reçoit ordre d'inhiber. La pudeur qui suit toute "Sortie $"$ altruiste dénonce la parole soumise à la passion et non à la raison: "Notre attitude à tous deux devait être ridicule, car nous sommes restés ainsi une bonne demi-minute, immobiles, la main dans la main [...] j'étais honteux de ma tirade; honteux d'avoir perdu pied ainsi. " (p. 53-54) II en découle une morale: l'esprit doit triompher du corps.

Prévenu contre la parole, Jodoin se réfugiera dans l'écriture. Qu'est cette écriture? Sans tromperie possible, elle est d'abord une écriture dont la précision, la rigueur et la recherche proclament explicitement son horreur d'une langue étriquée et implicitement son amour de la discipline. Elle est ensuite une écriture qui fait place à l'humour, un humour à double tranchant. On donnera à la première lame le nom d'humour qui passe par voie de la litote - comme son auteur - car il est à demi jeu de cache-ca- 
che. En effet, sous un faux air d'accessoire, tout contexte semble se prêter à l'attaque d'un tabou ou à la mise en lumière d'une vérité:

Ils s'approchent de moi avec des airs de conspirateurs et me glissent à l'oreille quelque titre ou nom d'auteur du ton dont on demande un condom ou un suppositoire chez le pharmacien. (p. 71)

C'était un jeudi après-midi, jour de congé au collège Saint-Roch qui se trouve [...] au milieu d'un vaste domaine appartenant à des pères qui allient l'industrie laitière à l'élevage des jeunes gens. (p. 74)

N'avez-vous pas un guide qui vous donne la cote morale des livres que vous vendez? s'enquit-il. Je lui ai répondu que je l'ignorais mais que, s'il voulait bien me donner le titre de ce livre de cotes, je consulterais nos listes. II a alors mentionné Le père Sagehomme et L'abbé Bethléem. (p. 79-80).

Cet humour-litote est omniprésent dans le journal et s'applique cunsciencieusement à franchir bien des interdits. Dans son deuxième visage, l'humour serait, à l'inverse du premier visage, hyperbolique car à la formule de l'aphorisme se substitue celle de l'emphase et du développement. Cet humour perce lorsque Jodoin entreprend de faire taire ceux qui oni droit de parole ou se l'octroient au pays de Québec, chaque contrainte au silence chez l'autre donnant lieu chez lui à un hors-d'cuuve verbal. On retrouve cet humour au niveau du rire insignifiant et de l'absence d'écho qui accueillent ses réparties à Nault (tranche du 10 mars); on le retrouve dans la froideur affichée durant les ergotages de Chicoine, laquelle met un frein à la discussion "sur les rapports de la morale et de la littérature (tranche du 17 mars) ou sème la confusion (tranches des 5 et 8 mai); il est dans la mauvaise foi dont Jodoin fait preuve dans ses réponses à sa logeuse ou au curé, lesquelles ont "quelque peu ralenti la faconde de Mme Bouthiller" (tranche du 7 avril).

A ce point, le lecteur observera que chaque imposition au silence favorise une élaboration qui se prête à la caricature d'un représentant de l'idéologie sociale prépondérante à l'époque. On s'interrogerà alors sur le sens du mariage humour et caricature. A cette interrogation, Dominique Noguez répond en répondant à la question:

D'où vient que l'humour répugne à la caricature [...] Car la caricature élimine et réduit, elle amoindrit [...] Si elle est "vérité criante», c'est de ce qui abaisse l'être, non de ce qui l'élève, de ce qui le perd, non de ce qui le sauve. L'humour au contraire [...] ne juge pas tant l'homme à cause de que malgré ses tares [...] l'humour est bienveillant et possède au plus haut point le sens de la solidarité ${ }^{26}$.

Que Jodoin se désolidarise de son prochain - mise en pratique de l'exclusion du précieux - les exemples foisonnent. Ajoutons, à ceux auparavant consignés, son mépris sans discernement à l'endroit des Joachinois en général qui entrent dans cette catégorie d' "abrutis qui attendaient dans la salle" de la gare (p. 106); ses plaintes contre les clients de la librairie, tous plus ou moins «crampons qui s'imaginent qu'[il est] là pour leur donner des renseignements, des consultations littéraires" (p. 35) ; ses précautions oratoires, les nombreux "peu importe" et les concessifs "comme on dit», qui établissent la distance entre la langue du narrateur et le parler 
de ses compatriotes. Cet homme à la parole châtiée est homme qui châtie autrui et celui qui refusait l'autre en lui-même refuse également autrui. Si d'une part il préserve de la sorte sa sensibilité à la fois contre cet autrui et contre lui-même, il provoque d'autre part la fermeture de son espace intérieur. Raideur stoïque, intolérance, soumission du corps à l'esprit, horizon intérieur et extérieur clos: l'univers de Jodoin est, grâce à sa lucidité, plus que cela mais il est parallèlement tout cela et, ce faisant, il ressemble étrangement au Québec traditionnel contesté. Double impasse et double paradoxe! En voulant projeter par le biais de son humour l'image d'un individu-contradiction des siens, le narrateur convoque un paraître qui dissimule que les structures mentales de son être foncier sont parallèles à celles de la collectivité à laquelle il appartient.

L'insensibilité de Jodoin est ainsi une demi-mesure. Elle l'est d'abord en raison de ses failles car il arrive à ce comédien, au cours d'un rôle, de vivre véritablement ses émotions. Fondée sur le postulat de l'impossibilité de communication, elle franchit trop souvent la distance entre soi et l'autre, se révélant infidèle à son idéal d'impassibilité. Elle l'est aussi parce qu'elle se découvre sur un plan essentialiste, par suite de ses résonances et de son recours à la contradiction, et malgré une intimité avec la satire, dédoublement des valeurs rejetées, ce qui l'institue tremplin d'une ambiguïté négative. Elle l'est enfin parce qu'elle interdit à Jodoin d'assumer un rôle de vie et le confine à rédiger un journal-évasion où ıl enregistre une situation de survie. La démesure dans ce contexte aurait été un cynisme sans défaillance ou, son contraire, une complicité sans calcul avec autrui. Jodoin aurait été, dans le premier cas, non le dilettante amateur mais le dilettante professionnel, heurtant par jeu les valeurs en place et sachant rompre avec elles dès qu'elles menacent l'intégrité de son insensibilité. Serait-ce qu'un arithmomane a fait d'insuffisants calculs? Dans le deuxième cas, la pudeur d'un Jodoin, plus humoristique que caustique, l'aurait cédé au lyrisme si bien que son espace intérieur et extérieur aurait été ouverture plutôt que circonscription et alors la voix directe, plus engageante, n'aurait pas craint de compromettre le raisonnable "tout est relatif" de la voix indirecte, de même que l'usage de l'hyperbole-libération aurait fait pendant à celui de la litote-censure:

L'humoriste reste le complice, "[/e] semblab/e, [/e] frère" de celui qu'il fustige. Pas de fossé comme entre l'ego triomphant et l'alter nihilisé de la condamnation. "Je suis comme vous, vous êtes comme moi": le «je» de l'auto-ironie rejoint le "vous" de l'ironie et suscite le «nous" humoristique ${ }^{27}$.

Que conclure sinon que l'heure de la présence, de la dialectique et de l'oscillation féconde du même vers l'autre n'a pas encore sonné: à s'intoxiquer de sel Safe-All, on échappe à l'ivresse puisque le dérangement en ce cas "se limite à un échauffement fort bénin". (p. 14) Mais, néanmoins, comme le paradoxe s'inscrit dans ce “déséquilibre" limité du narrateur, l'érosion des fondements d'un ordre social périmé s'en trouve accélérée, d'où s'explique en partie «comment la parole (écrite) vient au pays du silence ${ }^{28}$ "! 


\section{LES PÉDAGOGUES: YVES LAMBERT}

L'esthète Lambert évolue dans le cercle des pédagogues d'une école normale et parmi "le gratin de la société montréalaise" (p. 121), c'est dire au milieu de personnes professionnellement ou socialement mises en scène. Être pour autrui un point de mire est du reste un besoin vital chez lui, d'où le regard de cet autrui détient un rôle de première importance dans l'élaboration de son jeu. Il lui importe en effet de contempler dans les yeux des autres son image, non une image quelconque mais celle d'un moi idéalisé dont la moindre des exigences sera une apparence physique soignée. Cet impératif perce dès le début du récit et tout au long de ce dernier:

Lambert lissa du bout des doigts sa chevelure en panache. (p. 9) Lambert chassa d'une chiquenaude un brin de tabac collé au revers de son veston. (p. 10) Lambert s'approcha d'une petite glace près de la fenêtre pour ajuster son nœud papillon. (p. 11)

Du style, de l'ordre, de la beauté : le masque physique en sera un d'apparat.

Si une mise en forme extérieure s'avère essentielle, elle ne garantit cependant pas une acquisition spontanée de prestige. Au masque de surface doit se conjuguer un masque de savoir. Musicien de profession, Lambert cumule les fonctions d'enseignant et de critique musical, possède une licence ès lettres, falt figure de connaisseur en peinture, n'ignore pas l'art de la danse, pratique la rhétorique et s'exprime adans une langue étonnamment châtiée" ( $p .120$ ): bien qu'on ne puisse "nier qu'il y ait certaines correspondances entre les arts» (p. 144), il donne surtout l'impression, comme le consıgne son collègue Sigouin, d'être un de "ces dilettantes égarés en pédagogie" (p. 91). Mouvements sculptés et masque de savoir révèlent au fond la nécessité, chez ce musicien, d'orchestrer sa vie. II s'agit en tout temps et en tout lieu de composer chaque mot, chaque geste, chaque émotion comme il s'est agi d'organiser méticuleusement la pseudo improvisation-«interprétation audio-visuelle de quelques tableaux» (p. 124) à la soirée de la ministresse:

En réalité, la ministresse l'avait depuis longtemps pressenti au sujet de ce programme. Il avait accepté à condition que sa particıpation ne fût divulguée qu'à la dernière minute. Ainsi, croyait-1l, les invités penseraient qu'il s'agissait vraiment d'improvisations. II avait entre temps soigneusement pratiqué ses compositions chez lui. II les avait même enregistrées au magnétophone pour les juger d'une oreille objective. (p. 125)

La description de cette soirée, insérée au centre du roman, revêt une importance particulière pour la compréhension de Lambert puisque s'y juxtaposent les principaux éléments constitutifs de son univers. Dans un décor et une ambiance hautement dramatiques, on l'y retrouve en effet comme musicien, en compagnie de la femme qui a réussi à se tailler une place dans sa vie, Annabelle, et en présence des deux milieux entre lesquels se partage sa vie. L'atmosphère de pareille réunion élégante, imposant son rituel de convenances, s'avoue propice à l'épanouissement des tendances théâtrales, latentes chez certains, apparentes chez d'autres. Ces 
mêmes tendances s'accusent davantage chez les individus fascinés par les prestiges mondains et chez quı un désir de distinction s'allie au sentiment de la position hiérarchique. Ce désir fait qu'ils souhaitent se montrer reçus et considérés dans la haute société. Or, si sans en douter tel est Yves, il est intéressant et révélateur de constater que c'est par le biais des machinations de son double féminin, Annabelle, que le lecteur en vient à pénétrer les ressorts de sa personnalité sociale, laquelle se dilate lors de ce "raout de haute volée" (p. 122).

Quel reflet projette l'ex-ballerine française? C'est celui d'un esprit aventurier et arriviste, esprit qui découvre une femme manifestement préoccupée des apparences. À la source de cette préoccupation, une frustratıon: Annabelle, victime d'une sorte de malédiction après son refuge en terre canadienne, se sent rejetée du milieu qu'elle juge devoir être le sien:

Annabelle avait hanté pendant quelques semaines le bureau des impresarios dans le but de poursuivre à Montréal sa carrière de ballerine. Mais, soit malchance, soit manque de toilettes qui eussent mis sa beauté en valeur [...] elle n'avait rien pu trouver. Elle s'était dit que l'art de la danse au Canada n'intéressait personne. (p. 117)

Le succès au Canada de certaines comédiennes et chanteuses européennes au talent douteux et à qui elle devait sourire lorsqu'elles se présentaient à son studio avec des airs de reine la remplissait d'envie. Elle avait essayé de rivaliser avec elles sur le plan social, elle avait donné des thés [...] s'était liée tour à tour à un jeune chanteur canadien, à un impresario israélite [...] Pourtant, elle n'était pas satisfaite. Inconnue du grand public, privée du troupeau d'admirateurs [...] elle était admise dans ce milieu artistique comme une parente pauvre... (p. 118-119)

II en résulte un complexe d'infériorité qui la conduit à une cristallisation imaginative de la haute société montréalaise dont les membres se voient couronnés d'une auréole de magie. Cette cristallisation réfléchit un souhait de vivre sous le regard d'autrui et d'en être adulée, souhait qui semble constituer dans le cas d'Annabelle un prolongement de sa carrière de danseuse abruptement interrompue. Ainsi une insatisfaction, de caractère à la fois social, culturel et professionnel, provoque un besoin de styliser son existence et ce non pas dans la solitude mais au vu et au su des autres. Cependant, sous le fait d'embellir une réalité décevante et de remédier à des carences, se dissimule une faim qui est la faim d'illusions. Cette faim qui est douloureuse est aussi dangereuse: I'homme qui en souffre mutile son être au profit du paraître car il se trouve à la fois enchaîné au jugement d'autrui et lié par la puissance des leurres.

Que Lambert soit au même titre que sa maîtresse un illusionniste s'avère évident. Il est d'ailleurs éloquent que cette dernière perce sans hésitation son jeu lorsqu'il cherche à créer l'illusion que les manigances de la ministresse, pour l'emmener à s'exécuter au piano, l'ahurissent au plus haut point ${ }^{29}$. Se savoir deviné par une semblable ne l'empêchera néanmoins pas de jouer son personnage à l'intention de la galerie. Emboîtant le pas à la ministresse, l'acteur Lambert se montrera au cours de la fête artistique digne de son hôtesse et à la hauteur de sa préciosité mondaine. 
Mais, dès que l'on s'interroge sur les valeurs qu'incarnent la ministresse et le clan Deschambault, l'immobilisme et le conservatisme moral et social s'imposent d'emblée à l'esprit. L'indigence spirituelle d'une société fragmentée et pétrifiée s'étale avec complaisance, ce qu'illustre à merveille l'accueil du ministre au "cou encarcané dans son col montant ":

Le ministre [...] répétait inlassablement les deux mêmes formules de bienvenue: “Comment allez-vous, mon cher ou ma chère"; ou bien "Enchanté, monsieur ou madame", selon que la figure des arrivants lui parût ou non familière. Et en même temps, d'un geste incroyablement rapide qui contrastait avec la raideur de sa posture, il tendait une longue main osseuse aux veines saillantes. (p. 129)

Cette fête, qui représente le procès-verbal de l'idéologie bourgeoise et des personnages influents au Québec, forme le pendant du "tableau révoltant de notre système d'instruction publique" (p. 88) exposé dans les chapitres précédents. Et c'est dans ces deux milieux que s'écoule la vie de Lambert. II y a, d'une part, le gratin montréalais où Lambert est sûr de retrouver "Son cercle» composé “de sa pléiade habituelle d'admiratrices" (p. 155) et, d'autre part, l'École pédagogique, dépendante du clergé et aux «professeurs enchaînés, étouffés dans leurs aspirations, suant de peur à la pensée d'une mutation, d'un congédiement, vivotant à des traitements de famine" (p. 88). Ces ceux milieux proclament autant leur absurdité et nullité que leur vanité et monstruosité. Leur réunion à la soirée du ministre et de la ministresse en infère la symétrie, aussi leur appartenance à l'Identique se déclare sans équivoque:

Il y a deux puissances ici qui mènent tout: les politiciens et le clergé. Lesquels sont les plus forts? C'est assez difficile à dire. Parce que très souvent leurs intérêts coïncident. Et quand ils ont des différends, ça se règle presque toujours dans les coulisses. Le public n'en entend pas parler. En tout cas, ils se font des... concessions assez surprenantes parfois... (p. 209)

Il en ressort l'impossibilité d'une fécondation réciproque, d'où l'impossibilité d'un renouvellement.

Qu'il se situe dans une impasse, Lambert en est parfaitement conscient. L'insatisfaction qu'éprouve Annabelle est aussi sienne comme l'attestent ces considérations à Joyal relatives tour à tour à son pays:

- Je ne suis pas un touriste, dit Joyal. Le Canada, c'est mon pays.

- Moi aussi, dit Lambert. II n'y a pas là de quoi se vanter. (p. 62)

- On a beau dire qu'on n'est pas un touriste, on se sent un peu dépaysé quand on revient au Canada...

- C'est au moins une explication, dit Lambert. Moi je suis resté au Canada et je m'y sens comme un poisson dans l'air... Heureusement [...] j'ai appris à ne plus respirer. (p. 65)

à son travail :

Ces petits problèmes pédagogiques, moi, je m'en fiche comme de l'an quarante. Pour vous comme pour moi, j'en suis sûr, l'enseignement n'est qu'un job comme un autre. Ça laisse beaucoup de temps libre pendant l'année; et puis, il y a les grandes vacances. (p. 69)

à ses collègues et compatriotes: 
Plusieurs de nos instituteurs ont déjà commencé à singer les méthodes américaines. L'une en particulier consiste à enseigner la lecture sans passer par l'alphabet. Excellente façon, naturellement, de former des illettrés. Mais comme nos gens ne lisent tout de même pas, vous me direz que ça ne tire pas à conséquence... (p. 69)

Comme une ballerine s'est vue privée de son ballet, un musicien n'a pu ou n'a pas su s'épanouir dans la musique. La solution? Se réfugier dans les plaisirs mondains. Si ce refuge peut donner satisfaction au sens de l'esthétisme, il offre par contre à l'esprit créateur une bien piètre nourriture. L'artiste se révèle donc, par suite de sa dissémination, infidèle à sa vocation. Aussi, cet artiste sent-il s'élever peu à peu la voix de sa mauvaise conscience sous le regard de son collègue Joyal, jeune peintre au talent prometteur qui sait demeurer sensible aux problèmes d'un confrère désemparé et indifférent aux puissantes relations:

Si la crise à l'École pouvait se régler, Yves se promit qu'il allait faire un sérieux examen de conscience, apporter des changements à sa vie. Sans doute ne lui fallait-il plus songer à une carrière de musicien [...] [m]ais il n'était pas trop tard pour modifier sa vie. Pourquoi, par exemple, perdre son temps à papoter dans les salons [...] maintenant que les femmes du monde se disputaient l'honneur de sa présence, il se rendait compte que ça n'en avait pas valu la peine. Il continuait à jouer son rôle de bel esprit par vitesse acquise, par inertie [...] Seulement, voilà, était-il possible de réorienter sa vie à quarante-trois ans? (p. 248).

Moment de remords où Lambert découvre qu'il se gaspille en vaines activités et qu'il pèche contre lui-même en lésant sa vie intérieure. Mais le remords n'est que visiteur passager, il cédera de nouveau la place aux divertissements et aux faux-fuyants qui se chargeront bien de voiler le vide et d'ensevelir la mauvaise conscience. L'âge - les quarante-trois ans demeure-t-il la véritable pierre d'achoppement? À cette question, une réponse négative est fournie. On la trouve dans l'itinéraire divergent de l'ancien condisciple et actuel collègue, Sarto Pellerin, lequel réoriente après ses quarante ans sa vie en accord avec son être véritable. Lambert, au contraire, oppose un refus et opte pour la survivance de son être d'emprunt: il continuera de mener "la vie la plus bourgeoise du monde" et ne quittera pas "une situation de tout repos qui [lui] laisse beaucoup de temps libre" (p. 247). Au psychologue Sigouin revient d'avoir eu raison en incluant Yves parmi ces hommes "qui se montraient les plus réfractaires aux in: novations" (p. 93).

Devant cette résistance-pétrification, non dépourvue d'affinités avec "la peur béotienne" caractéristique des Pédagogues, et face à la démesure de Pellerin, on ne peut conclure qu'à la demi-mesure de Lambert. Il côtoie sur sa route toutes les possibilités mais toujours il les répudie ou les occulte. Il y a ainsi la possibilité d'être musicien mais pourquoi ne pas se contenter du "simple pianotage d'amateur" (p. 65); il y a celle d'être un véritable ami mais n'est-il pas plus prudent de recourir à la diplomatie d'une "'petite intervention' auprès de la ministresse" pour régler les "petits emmerdements de ce cher Sarto» (p. 207), même si Lambert sait que ce der- 
nier refuse le compromis et espère son appui moral; il y a la possibilité d'être mari mais cette condition n'égale pas «un certain plaisir à jouer son rôle de parti désirable et récalcitrant, faussement naïf [car] ces efforts que l'on faisait pour 'l'attraper' lui prouvaient son importance [...] Une fois marié, c'étaient là de petites douceurs dont il devrait se passer " (p. 249-250); il y a celle d'être père spirituel mais ne vaut-il pas mieux séduire l'imagination de l'enfant en dressant de sa personne un portrait avantageux, quitte à rater son coup et à perdre sa postérité : «il surprit dans le miroir l'expression dédaigneuse de Joyal qui, le coin des lèvres rabaissé, branlait la tête. 'Il me méprise'. Cette pensée le piqua au vif" (p. 210). Il y a enfin la possibilité d'être compagnon pour gens de son sexe, mais n'est-il pas plus gratifiant et moins exigeant d'être l'idole d'un cercle d'admiratrices:

Les hommes étaient assez clairsemés. Ils se rendaient compte que le pianiste n'attachait pas d'importance à leur compagnie. Son continuel persiflage, ses boutades déroutantes au milieu d'une discussion sérieuse leur déplaisaient, les désarçonnaient. (p. 155).

La pétrification de la société sollicitait un renversement. Lambert toutefois, en se repliant sur soi, s'est interdit de jouer le rôle de chef d'orchestre auquel semblait symboliquement le désigner sa formation de musicien. Pourtant, il portait en lui les germes d'une démesure qui lui aurait permis d'harmoniser les voix discordantes de son entourage: "voix imperceptible" de Sigouin, "voix éteinte" de l'abbé Béchard, "voix de sauterelle" ou "voix grinçante" de Sarto, "voix solennelle" d'Arthur et de la ministresse, "voix croassante" du ministre, "voix sifflante" de Sloper, "voix coupante" ou "voix de fausset " de Marcotte. Aussi, à celui qui s'oppose à jouer un rôle décoratif et dont la voix peut devenir passionnée, à ce "Joyal [qui] ne se sentit pas le droit de se défiler" (p. 144) revient le mot de la fin, mot qui, parce qu'il est ouverture, porte l'espoir d'une révolution de l'être et de sa rédemption: "L'art est avant tout communićation." (p. 144)

\section{L'INCUBATION: WEINGERTER}

C'est dans un espace de distance sans communication qu'est enfermé Weingerter, "l'antique pédagogue teuton bibliophage» (p. 46). Cet espace est l'espace de la mort, comme l'insinuent l'impuissance du philologue et son âge avancé, et déclenche la fuite de la vie, comme l'indiquent l'étiolement à ses côtés de Néa et le suicide de celle-ci. Weingerter, cependant, se révèle un homme qui fuit la mort, autant la sienne par ses 'éternelles' recherches sur la dérivation onomatopéique que celle de Néa dont la vie devient garantie de la survie de Sara, l'épouse disparue. Comment le masque de la mort en est-il alors venu à épouser les traits du dramatique érudit?

Weingerter est au départ un professeur, un universitaire. II l'est non dans la manière de "ce clown ignorantin ce minus habens de Blackwell (à Vienne ach à l'université de Vienne il eût été tout au plus concierge) [...] un pitre du dernier acabit"( $p .72$ ), ni dans le style de "cet hurluberlu de Ripcord " qui “à Vienne [...] aurait été tout au plus jockey, même pas: 
garçon d'écurie bei uns à Vienne: pelleteur de fumier» (p. 144); il est, lui, “Herr Professor Wilhelm Richard Bartolimeus Weingerter» (p. 71), spécialiste de "la dérivation onomatopéique comparée dans les langues indoeuropéennes" (p. 119). Dès lors, il se range au nombre des esprits supérieurs et ce faisant se distingue non seulement des professeurs qui, à son avis, "ici n'en menaient pas large" (p. 61) mais aussi des habitants, jeunes ou vieux, de son pays d'adoption:

on n'avait pas idée ici en Amérique dans un pays comme le Canada, il le disait en toute amitié, mal dégrossi, où les cerveaux dans des espèces de limbes.../ portant un dénonciateur arthritique index vers les vieillards lézardeux assis sur leurs bancs/ (p. 117).

Correspondre à l'image du «herr professor " est l'idéal que Weingerter n'a jamais cessé de poursuivre et poursuivra sans cesse. Déjà, dans le lointain passé, il avait fait de cet idéal son dieu: "écoutez, moi à Paris à Heidelberg à Vienne, quand j'étais étudiant puis Privatdozent, seize heures par jour six jours par semaine: Arbeit Arbeit Arbeit, à la force du poignet voyezvous bien, une concurrence féroce sans merci » (p. 118). Octogénaire exilé à Narcotown, il y demeure fidèle: a avec toutes ces recherches ces travaux il faudrait vivre jusqu'à cent ans et ne pas dormir du tout" (p. 114). Entre les deux, il y a eu Sara et l'amour, la guerre, la disparition de l'épouse, l'exil, l'apparition de Néa mais, au-delà de tout et dominant tout, il y a un puissant pouvoir, "toute sa science linguistique philologique morphologique" (p. 114). S'il y avait, selon le mot de Rabelais, un abime de science, il y avait aussi une science qui creusait un abîme car l'être qui la possédait lui sacrifiait tout, se contentant de vivre «inconsciemment myopement métaphoriquement». C'est ainsi depuis de longues années que cheminait l'incubation d'un mal, un mal qui se rattache à la maladie de l'idéalisme et à un certain refus de la vie. En effet, dès le moment où Weingerter a choisi d'œuvrer dans le professorat et la recherche, il consacrera ses efforts à réaliser jusqu'à la perfection cet idéal dont la solidité n'a d'égal que l'espace dans lequel s'exercera sa profession: "remontant [...] jusqu'à cette université imperméable [...] rassurante par la massivité même par la raideur même de son hostilité" (p. 116). Comme l'erreur est défendue, seules trouveront place les obligations réglées sur la fin. Nulle surprise que Maggie, dont le foyer se distinguait «à la belle impeccable inattaquable ordonnance" (p. 108), trouve grâce à ses yeux! Ainsi, une contrainte intérieure est appelée à métamorphoser en obstacle ce qui lui est extérieur, d'où s'identifie-t-elle à l'emprisonnement.

Dans cet espace clos, autrui se posera en intrus et la première victime de cet idéaliste sera l'être le plus près de lui, l'être qui menace le plus son intégrité, sa femme Sara. Celle-ci avait pourtant été jadis, à l'âge de l'impétuosité, sujet de transgression puisqu'il n'avait pas craint “d'épouser par amour (un amour attisé exacerbé par la résistance des deux familles) Sara une juive de s'installer (par bravade ou par un obscur sentiment qu'ailleurs ç'aurait été pour Sara plus difficile, pour lui plus irritant) dans la Berggasse la freudienne Berggasse [...] de s'installer donc avec Sara (qu'il aimait qu'il avait conquise de haute lutte qu'il aimait qui était pourtant déjà sujette 
à, quii donnait déjà certains signes de) dans ce demi-ghetto..." (p. 116-117). La force de la compétition se le dispute de toute évidence à celle de l'amour et l'une aura préséance sur l'autre comme le corroborent ces réflexions ultérieures de Weingerter:

on vit ensemble on se parle se voit tous les jours on partage la même table le même lit on va on vient on a de l'ambition on veut arriver on se dit inconsciemment myopement: la réputation la renommée que je m'efforce que je suis en train d'acquérir va quand même va rejaillir sur elle (qui est moi qui fait partie de moi) lui procurer le même sentiment de satisfaction... (p. 119)

La parenthèse est ici très révélatrice car, constat d'une appropriation, elle dénonce le narcissisme du savant méconnaissant la différence pour ne reconnaitre que son propre reflet. Faisant lui celle qui est autre, plus rien ne l'oblige dorénavant à laisser vivre en lui cet autre qui existait au moins virtuellement avant qu'il ne devienne sien : il signe ainsi sa propre condamnation à demeurer perpétuellement le même.

Pas plus que l'autre n'avait droit à la vie, n'aura-t-il droit à sa mort. Le corps de Sara n'est certes plus car elle "avait péri, absorbée étiolée décomposée dans ce germanique enclos concentrationnaire derrière des barbelés" ( $p .119$ ), toutefois l'inanition de Sara n'entraine pas chez Weingerter la consommation de la rupture. En effet, il éprouve encore à plusieurs dizaines d'années de distance le besoin du double et substitue Antinéa (Néa) à Sara. Bien peu l'inquiète leur impossibilité de communication comme bien peu lui importe l'indifférence de Néa, qui traîne ici-bas une misérable existence, à l'endroit de cette vie! Weingerter l'oblige à survivre car il attend d'elle la disculpation et une communion jadis ratée avec Sara:

Antinéa et Weingerter étaient des poissons ach des crustacées flottant immobiles repliés involués sur soi, incapables de se toucher rejoindre [...] émettant un certain nombre de paroles de propos décousus incousus, il éprouvait pour elle Menschenskinder une affection profonde paternelle, cherchant malgré tout à l'atteindre la dénouer la soulager mais toujours impuissant inefficace, mein Gott, Dieu sait s'il avait pourtant l'habitude dans les livres les manuscrits [...] de dépister dénicher des signes des indices, de flairer subodorer la petite phrase significative la particularité stylistique le tic révélateur. (p. 70).

En ne lui accordant pas ainsi la permission de partir, il souhaite au fond ressusciter cette partie de lui-même, toujours répudiée, qui se rattache à la vie affective. Cependant, il y a risque, à ne cultiver que la vie intellectuelle au détriment du reste, que l'hypertrophie du cerveau atteigne à quatrevingts ans une telle démesure qu'elle devienne un mal incurable. Abrité dans un "sanctum sanctorum philologique" (p. 143), celui qui en est frappé, perdu dans ses "radotages ruminations phobies" (p. 115), ne demeure capable à l'égard d'autrui que de demi-mesure puisqu'il demeure, au présent comme au passé, incapable de "prévoir les dangers les écueils, naviguer entre les récifs" (p. 123). II y a ainsi dans le présent par rapport au passé une symétrie de situations et une répétition stérile parce que porteuse des mêmes erreurs. 
Face à l'acteur qui se dépense sur la scène, Weingerter ne saura être que spectateur, regardant agir en évitant d'intervenir. Mais, son regard est celui d'un myope, d'où est-il à courte vue et non à double vue. Ne dit-il pas lui-même: "la plupart des hommes sont aveugles comme des taupes qui ne voudraient pas voir, qui ne se donnent même pas la peine d'essayer ach de s'ouvrir les yeux" (p. 122) et à ces hommes il se joint implicitement en poursuivant: "prenez par exemple a case in point : la dérivation onomatopéique" (p. 122). Une "dérivation» intellectuelle mène à une dérive de l'être et l'«affection profonde paternelle» envers Néa ne sera qu'une demiaffection comme est une demi-vénération la vénération témoignée à l'égard de Sara: "Ça (i.e. l'arrivée de Néa) avait non pas peut-être bouleversé (c'était un bien gros mot) mais comment dire resensibilisé sa vie» (p. 63). Dans les deux cas, Weingerter se contente d'une parodie de l'amour, sorte de jeu du hasard, d'où il joue "métaphoriquement", à l'instar de Maggie et de ses "thés marivaudants", une comédie qui est vouée à demeurer la comédie de l'onomatopée. Ainsi l'insinuent les multiples sons qu'il émet sans cesse: "se mouchant s'ébrouant" ( $p$. 117), "ébranlé secoué terrassé d'un sismique inextinguible rire [...] toussant strangulé postillonnant, les crises recommençant renaissant après des pauses laborieusement consacrées à de rauques inspirations" (p. 144-145).

Cependant, parce que le demi est dans l'incubation la mesure de chacun des personnages qui tous vivent « une demi-vie» (p. 53), " circulant à la surface du sol dans un demi-rêve" ( $p$. 150) "à mi-chemin entre la veille et le sommeil" ( $p .20)$, leur vie kafkaesque ne camoufle-t-elle pas cette comédie dont Gordon dit qu'elle est “du goddam sous-Marivaux" (p. 154) et à propos de laquelle il conclut: “comme comédie c'est raté, un four» (p. 154)?

\section{LE CYCLE: JULIEN}

Qu'un jeune rebelle se réfère à l'expression de son amour ou à ses aspirations révolutionnaires, une idée demeure sous-jacente: celle de combat. Ce combat est un combat qui se livre dans l'ambivalence car le protagoniste, se donnant l'illusion de poursuivre l'érotisme au niveau de l'amour et le socialisme au niveau de la lutte, escamote sans cesse le corps à corps pour trouver asile dans l'utopie. II est à ce sujet caractéristique que son combat se pare d'un masque religieux et se perde dans une étourdissante faconde: religion et verbe chercheront à provoquer le feu incendiaire qu'une sexualité s'avère impuissante à déclencher, d'où la luxure s'avoue chez lui plus cérébrale que sensuelle, le plaisir plus épidermique que passionné.

La nouvelle religion de Julien se voudrait profanation et renversement d'images, rites et symboles traditionnellement sacrés mais n'arrive qu'à être dérivation de l'ancienne religion dont elle prolonge de ce fait la survivance. On y décèle ainsi le culte du père lointain tout-puissant, lequel emprunte les traits de Stanislas-Auguste Casavant - «chef souverain [...] 
moulé dans un survêtement noir" (p. 81) - et celui de la mère bienveillante et protectrice, laquelle épouse la forme de Sophie Teunebröker «merveilleuse merveilleuse Sophie internationale se dévouant ici luttant ici..." (p. 85). Cette religion inclura même le raffinement de l'institution parvenue à une certaine évolution puisque la lettre $X$, l'allusion historique et le symbole du triangle sont associés à ces divins Père et Mère: "le chef [...] bras obliquement brandis formant un $X$ indestructible et dynamique [...] la pyramide pharaonique inébranlable des jambes du chef [...] triangle isocèle des jambes [...] triangle inversé pelvien soyeux vertigineux de Sophie" (p. 84). De plus, l'observation du rituel de vie d'un anarchiste libertaire, lequel préfère le "galetas miteux", l' "antique matelas hernieux ramassé dans une ruelle " et les "couvertures trouées de la Salvation Army" à "l'avachissement bourgeois" ( $p .85$ ), n'est pas sans rappeler l'idéal de vie austère mis en pratique par les religieux: Julien ne qualifie-t-il pas Sophie et lui d'، authentiques ascètes d'une imminente renaissance" (p. 85)! Mais, voudrait-on comme Roberto, l'amant de Berthe, lui faire préciser l'efficacité de sa participation à cette régénération ou donner une réponse satisfaisante à l'interrogation de sa mère Vitaline, "Qu'est-ce que ça veut dire nationalisme socialisme séparatisme droit à l'auto-détermination damnés de la terre nègres blancs levez-vous qu'est-ce que ça veut dire" (p. 45), on se heurtera à un disciple "se gargarisant de formules creuses" (p. 72) et dont la voix se perd dans le concert des "gosiers calvairiens et hostiaques hurl[ant] leur foi nouvelle" ( $p .93$ ) :

Père qui vis en moi qui palpites en moi au sein de la nouvelle Supertribu nous détruirons ensemble le vieux système pourrissant comme un quartier de buffle nous ferons éclater la séculaire cellule triadique nous projetterons d'unanimes et universelles constellations. ( $p .93$ )

Julien choisit ainsi, conformément à un idéal que la mode et le temps proposent aux jeunes intellectuels, d'être un révolutionnaire. Or, "garçon naguère sensible" (p. 30), surprotégé et encore fragile, il n'a pas la trempe pour bien camper ce personnage. II a beau s'évertuer à en épouser les apparences - «élocution parlure joualisantes [...] ayant renoncé à tout au confort au diplôme universitaire à la niche sociale assurée» (p. 33), "errant au milieu d'un troupeau de barbus narcomanes et de filles malpropres aux crins poisseux hantant les meetings les cellules marxistes séparatistes maoïstes socialistes" (p. 34) - il n'hérite pas pour autant de l'état d'esprit qui donne naissance à l'authentique révolutionnaire. Qu'il chante et joue faux nous est indiqué par sa sœur Gaétane, jadis béate admiratrice de son frère, qui maintenant superpose constamment les faits et dires de ce dernier à ceux de son professeur d'anglais, "l'impeccable" et fascinante Mademoiselle Lacoste, locataire d'un «penthouse sur la montagne» (p. 34), qui de sa "voix calme et ferme" (p. 34) met ainsi ses "girls" en garde: "If you are ever accosted by young bums or beatniks - as they are now called - don't answer don't even turn your head. That would be reducing yourselves to their level. " (p. 34)

Pourquoi une telle faille chez Julien? Tout simplement parce que ses choix et comportement de révolutionnaire relèvent d'un idéal factice puis- 
que liés de fait à l' «antique implacable CEdipe antéconscienciel ${ }^{30}$ "(p. 94). Cet idéal est la prolongation des jeux d'enfance durant lesquels Julien, monté sur sa "jument Sophronie ${ }^{31}$ " aux "sabots pétaradants", "sous les yeux extatiques de sujets tremblants", mimait et se racontait comment il poursuivait et exterminait “le tyran monstrueux le barbare étranger»qui lui avait usurpé son royaume (p. 86). L'adhésion révolutionnaire s'avoue dans ce contexte illusion et refuge, d'où Julien a besoin, pour percevoir son être révolutionnaire, de s'abreuver de clichés et de se dissoudre dans un décor et une atmosphère dramatiques:

Familles bourgeoises je vous hais capitalisme je te maudis société de consommation je te dégobille de toutes mes tripes système infâme... (p. 98)

déploiement spectaculaire interminable attente drapeaux fleurdelisés plate-forme surplombante et dominatrice fanfare aux musiciens écarlates [...] réflecteurs violacés [...] au moment stratégique comme un coup de tonnerre fusant des haut-parleurs tronçoniques éclatera notre hymne de ralliement [...] puis déjà dans le faisceau aveuglant des lumières [le chef] sera là [...] je crierai hurlerai de toute la force de mes poumons. (p. 89-90)

Voilà un socialisme qui s'orchestre, à l'instar de ses conquêtes enfantines, sur de bien bruyants tambours! Aussi, le feu sacré semble voué, "dans cette cornue grouillante chauffée à blanc" (p. 89), à se volatiliser en feu d'artifice.

Le jeu de Julien, s'il est théâtral; est aussi narcissique. Narcissique non seulement parce que le jeune homme se perd "dans la contemplation de [son] nombril» (p. 91), mais aussi parce que ce faisant il cherche à dresser de lui-même un portrait avantageux dans l'imagination de Sophie. Mais comme cette Sophie tient à la fois de sa mère, de l'idole et de la rivale, il se fourvoie en voulant atteindre ce but. En effet, pour être à la hauteur de la "stoïque", "l'incorruptible l'inébranlable» (p. 84) Sophie, il faut pouvoir franchir spirituellement l'interdit social et moral. Julien, pour sa part, le franchit fictivement au moyen d'une pose et de symboles: jouant à l'anticonformiste cynique, il s'habillera, parlera et vivra en conséquence. A contester une réalité opprimante par l'affectation et à réfuter une aliénation par le symbole, Julien dispute sur un plan extérieur un combat intérieur. II n'y a ainsi rien d'étonnant à ce qu'il soit toujours pris entre deux feux, tantôt aiguisant en lui-même de multiples désirs sans jamais réussir à les assouvir, tantôt exaspérant ses sens sans parvenir à les satisfaire; ou encore à ce qu'il soit en proie à la contradiction car si, d'une part, il cherche avec "self-control et méthode" (p. 83) à s'intégrer à la jeunesse qui bafoue la morale conservatrice, il succombe, d'autre part, aux "catacombes passéistes" (p. 105) et à l'emprise familiale: “comment puis-je ne pas avoir de passé n'ai-je pas eu des parents un frère des sœurs une enfance à la maison une adolescence un bout de jeunesse" ( $p$. 103-104). Révolté trop chargé affectivement, il n'est, au moment d'engager le combat, qu'un révolutionnaire mal armé, marchant vers l'échec puisque sa révolution sociale exigeait d'être précédée d'une révolution psychique. 
Parce que son jeu demeure à ses yeux interdit et que son masque se lève sur une absence, ce jeu devient dangereux. Julien élude par ses divers alibis et faux-fuyants la possibilité de communication, de fusion, de coïncidence avec autrui. II n'y a donc aucune chance que sa relation avec l'étrangère Sophie Teunebröker ou celle avec la foule "des nègres blancs porteurs d'eau messianiques damnés de Terre-Québec" (p. 99) se développent en relation d'appartenance. Dominées par l'absence d'identité et un sexe où règne la disjonction corps/esprit, ces relations se teintent de masochisme et se découvrent auto-mutilation. Mis en pareille situation, le bourreau-autrui ne laissera jamais tomber, pour se livrer à sa victime, ce masque qui lui permet d'agrandir sa taille et de renforcer sa voix. Et ainsi, faute d'affranchissement, le jeu de Julien se perd dans les mots : si l'amplification oratoire crée passagèrement l'illusion de combler une absence par un effet de présence, elle ne tarde pas à signifier qu'elle dissimule un être de carence. Chez cet être, le masque révolutionnaire coïncide avec "ce masque rigide figé " (p. 92), si souvent évoqué, du père, cet a irrécupérable ". C'est là une image dont la répétition est d'autant plus obsédante que ce géniteur mort, désormais enfermé dans l'espace clos de son "container", porte inscrit le cheminement et la dissolution de son fils dont l'aliénation musèle la propre violence.

Si la rébellion de Julien s'articulait sur une passion authentique plutôt que "claustrophobique", elle ne se disperserait pas en une série de clameurs isolées, ni ne dégénérerait en nausée mais, hantant les profondeurs, elle transformerait son délire verbal en délire libérateur. II ne serait plus question de vomir de grands mots, visant à décupler de maigres sensations, ou de hurler en chœur de faux cris, invoquant une vague fête collective à venir, mais de traduire une abondance ou plénitude vitale qui refléterait une conjonction du corps et de l'esprit, une conjonction de l'homme et de son masque ainsi qu'une conjonction de la passion individuelle et communautaire. Ce serait l'adhésion à une nouvelle religion et non fidélité à la norme de l'ancienne, car Julien passerait alors par la communion et la transfiguration de la démesure révolutionnaire et non par la négation et l'exaspération de la demi-mesure du précieux à rebours.

Au moment de résumer les lignes de force qui se dégagent des propos sur les précieux, il vient d'abord à l'esprit qu'on peut les considérer comme un personnage unique à facettes. Comme il est du reste possible d'opérer le rapprochement Augustin/Julien, puisque les traits partagés d'un même âge, de l'CEdipe, de la verbosité, de la mauvaise foi et de la mauvaise conscience y incitent, la réunion des précieux évoque dès lors l'idée d'évolution de ce personnage. Elle renvoie en effet tour à tour aux stades de la jeunesse (Augustin, Julien), de la maturité (Jodoin, Lambert) et de la vieillesse (Weingerter).

L'idée du personnage unique éveille celle du fonds identique, ce qui amène à. consigner les caractères communs aux divers précieux. Parmi ces caractères, c'est au premier chef leur aspect théâtral qui s'impose. Cet aspect signale l'importance du masque, lequel suppose le désir de simuler 
afin de dissimuler. Ce que l'on veut ici simuler, c'est la présence du plein et ce afin de mieux dissimuler le vide d'une absence foncière, celle de l'amour. En deuxième lieu ressort la dépendance du précieux à l'égard d'autrui qui seul lui permet de se définir: le sort du comédien apparaît lié à la réaction de l'auditoire. Les relations qu'il développe avec les autres personnages s'insèrent alors dans un rapport de complémentairté ou d'opposition. Lorsque le rapport d'opposition prévaut, la complexité entre en jeu car il ne s'agit plus du simple affrontement de deux individus mais du combat à mort d'un idéaliste aux prises avec un individu/société réactionnaire qui cherche à l'avaler. La disjonction se découvre donc inhérente à cette opposition. Cette dernière présume à son tour une distanciation de la société québécoise, laquelle incarne une réalité inacceptable et ce faisant prive l'être de l'espoir de communication.

A l'aspect théâtral et à la disjonction avec autrui se conjugue l'impuissance du précieux. Impuissance entachée de culpabilité par suite de sa filiation avec un sexe où corps et âme sont dissociés au lieu d'être associés. Impuissance qui en outre s'accompagne d'un sentiment d'échec puisque l'être lésé ne débouche pas sur un engagement personnel mais se réfugie dans un beau langage et le raffinement intellectuel. II devient ainsi évident que la préciosité est chez ces personnages la forme qui répond à un besoin psychique. Convergence d'un fond vers sa forme, le langage des précieux s'offre comme un jeu d'échos et d'équivalences d'un être indissociable du paraître: il est miroir. Et ce miroir des mots obéit chez eux au principe de la richesse et de la prolifération. C'est le règne de la recherche mais aussi de l'hyperbole où les mots sont biens de consommation et de dissipation. Tout se passe comme si le précieux réagissait par la surenchère verbale à l'assassinat de l'individu par la société, lequel se double dans le milieu québécois où il évolue d'un assassinat collectif du corps et de la langue. Ainsi, la préciosité ne saurait dans cette perspective s'isoler de l'aliénation, aussi est-il des plus révélateurs que le précieux se dévoile dans chaque cas un être limité et privé de lieu. Ces remarques éclaireront dès lors que s'observe autant chez Augustin et Jodoin que chez Lambert, Weingerter et Julien l'existence d'une demi-mesure au niveau de l'échange avec autrui qui a sa contrepartie dans une démesure au niveau đu verbe: l'écart d'ordre affectif cherche à être comblé mais, comme l'équilibre instauré est d'ordre intellectuel, il demeure aussi menaçant et menacé que celui du funambule!

Si une langue riche et la recherche formelle obéissent chez les précieux de Bessette à une nécessité psychologique, elles découvrent chez leur auteur une exigence artistique. Aussi, alors que les créatures continueront de faire figure d'artistes ratés, le créateur projettera-t-il au fur et à mesure que progresse son œuvre l'image du maître-artiste. Puisque les constantes précieuses vont s'accentuant et s'affinant d'un roman à l'autre $^{32}$, la demi-mesure qui se situait au point de départ de la Bagarre se transforme au point d'arrivée du Cycle en démesure après être passée par une série de variations dont la première, le Libraire, épousait, par suite de 
la condensation de son intrigue et de son écriture serrée, un mouvement ascendant, la deuxième, les Pédagogues, en raison de la dilution de son intrigue et de son écriture calculée, un mouvement descendant tandis que la troisième, l'Incubation, provoquait, avec la fragmentation de son intrigue et son écriture délirante, l'éclatement de la "mesure». Et c'est ainsi que les tirades éblouissantes d'un dilettante dans le roman linéaire au début ont dans le roman baroque de la fin cédé la parole aux introspections tout en entrelacs de sept personnages, substituant de la sorte une véritable architecture verbale aux simples saillies et jeux d'esprit d'Augustin Sillery. Mais, c'est d'abord dans l'Incubation qu'avait fait son apparition cette alliance de la technique et de l'art telle qu'on la retrouve dans le Cycle. Cette alliance s'y manifestait dans l'organisation rigoureuse, au niveau de la narration, de l'apparente "trame déchiquetée", grâce à l'adresse des transitions basées sur un système de ponctuation original et anti-conventionnel. On l'y percevait également, au niveau du style, dans cette richesse qui résultait du retour de métaphores prolongées et de l'emploi réfléchi d'une variété de procédés: phrase sans coordination répondant au jeu de libre association des personnages, énumération, répétition, séries synonymiques, invention verbale et recours au participe présent. Bessette trouvait, en mettant ainsi en œuvre ces divers moyens, la forme qui calquait le continuum psychique d'un narrateur alcoolique dont le récit était soumis à d'incessantes ruptures de chronologie.

Que la préciosité marque de son sceau l'Incubation s'avère probant autant parce que le roman baigne dans l'artifice, la rhétorique et la recherche que parce que divers types précieux - dandy, snob, maniériste, décadent $-y$ sont représentés en Ripcord, Maggie, Weingerter et l'érotomane torontois. Ce lien avec la préciosité s'avoue encore dans le Cycle. L'aveu y est même en quelque sorte littéral puisque l'un des personnages invite, en rappelant l'Astrée d'Urfé et la Carte de Tendre de Mlle de Scudéry, à associer l'interminable voyage dans les profondeurs de sept personnages au parcours complexe et compliqué du Pays de Tendre. N'est-il pas du reste éloquent qu'aujourd'hui comme hier la psychologie préside aux destinées du périple puisque c'est sur le schème freudien de l'organisation tripartite de l'appareil psychique que s'ébauche le dernier roman? Le raffinement est aussi poussé maintenant qu'autrefois et preuve en est que le rythme du passage où sont insérés les "précieuses" allusions littéraires reproduit, en accord avec les gestes et pensées de Berthe, les diverses phases de l'acte sexuel ${ }^{33}$. La relation entre fond et forme est à ce point heureuse dans le Cycle qu'il est fréquent de voir le rythme se modeler sur les concepts freudiens maniés par l'auteur tels pulsion, condensation, équilibre, brouillage, effervescence.

On ne saurait s'empêcher de songer devant cette analogie du corps physique et de l'écriture à la réflexion de Sollers: “Le sexe et l'écriture sont liés de telle façon que l'un est sans cesse la métaphore de l'autre [...]. C'est là le lieu d'un renversement constant ${ }^{34}$. "L'importance que prend le sexe dans l'Incubation et le Cycle paraît confirmer les dires de Sollers. 
D'ailleurs, comme Bessette a lui-même inscrit la connotation sexuelle de la langue châtiée ${ }^{35}$, langue que son écriture fait sienne, il vient à l'esprit, lorsque l'on cherche une approximation de la préciosité considérée dans son ensemble, d'adapter le célèbre mot du Neveu de Rameau en: "Mes paroles, ce sont mes catins."

Appendice ${ }^{36}$

\section{LA COMMENSALE: JÉRÓME CHAYER} (p. 124).

"[N]ous étions des âmes sœurs, nous macérions dans le même jus"

Si c'est une compatibilité de caractère avec Athanase Bessière qui inspire cette réflexion au protagoniste de la Commensale, Jérôme Chayer, cette réflexion nous inspire en retour un rapprochement entre le libraire Hervé Jodoin et le comptable Jérôme Chayer. Les ressemblances sont en effet nombreuses entre ces deux arithmomanes-humoristes, "masturbateurs intellectuels" (p. 154) qui rédigent le journal d'une tranche de vie pour tuer le temps, aussi ce qui a été dit du premier s'applique au deuxiè$\mathrm{me}^{37}$.

Sous les multiples affinités de ces “âmes sœurs" percent cependant certaines différences. La plus frappante se situe au niveau du degré d'intensité. Actions, pensées, paroles, sentiments de Chayer sont, comparativement à ceux de Jodoin, affectés du coefficient "plus»: Chayer n'inscrit-il pas "les additions" comme "soupape" numéro un "sur la liste de [s]es tue-temps " (p. 154) ! II résulte curieusement de cet état de fait que si Chayer, nature absolue, est un être plus monstrueux, plus démesuré que Jodoin, nature relative, il est concurremment un être plus humain, plus mesuré: son besoin d'autrui - Paulo, filles des lupanars, Madame Bessière, Athanase - en témoigne, même si ce. besoin doit nécessairement, pour demeurer digne d'auto-estime, se doubler du mépris de cet autrui. Chez Jérôme, le fond ne va pas sans «le frappant de la forme» (p. 120)!

Chez ce même Jérôme se discerne un formalisme absent chez Jodoin, ou du moins beaucoup plus effacé. Ce formalisme se traduit tantôt dans un souhait de "faire les choses en grand" et d' "impressionner Madame Bessière (p. 19) par une invitation à manger au Coq d'or avant de procéder à la séduction, tantôt dans la volonté de soumettre à une norme implicite les relations avec son directeur et ses collègues de bureau. C'est encore lui qui amènera Chayer à s'astreindre à l'observation de certains préceptes qu'il a lui-même établis: ne jamais déjeuner seul pour considérations d'ordre gastrique, ne pas fumer le cigare dans la matinée pour question d'habitude, se conformer à un rituel précis pour fins de concupiscence, circuler avec trois montres pour continuelle vérification à la minute près, préférer les chiffres aux mots pour "protection optima contre les désillusions" (p. 123), manier “l'injure avec une certaine dextérité " (p. 86) pour 
“déboulonner avec méthode et précision» (p. 123)... En tout et partout le comptable tient ses comptes: une dépense de soi ne va pas sans une diligente rentrée-compensation, ainsi l'exige le bon fonctionnement d'un auto-évaluateur, «machine à calculer» (p. 123) mécanique sinon maniaque.

Bien qu'enclin aux excès mesurés, un terrain demeure où Chayer se formalisera à outrance, le terrain linguistique. Ainsi, ne ratant jamais l'occasion de s'attaquer à la pauvreté, à l'imprécision, à l'inarticulation et à l'anglicisation du parler québécois ${ }^{38}$, il ne restera pas davantage, bien qu'il affirme n'être "pourtant pas puriste" (p. 110), celle de rectifier l'erreur ou de combler l'ignorance, à savoir l'«acception abusive» qu'un Paulo ivremort, à la démarche flageolante, prête au "vocable marcher» (p. 102), ou son audace à "parler de 'coup de pied au cul' et 'd'aplomb' ” pour signifier «l'attouchement de l'extrémité de son couvre-chaussure et de la région lombaire inférieure du patron" ( $p$. 116), à savoir encore les commentaires didactiques relatifs aux "libertés morphologiques" que se permet un de Repentigny acculé à un accès de rage (p. 109-110). Trêve de dégénérescence: le moindre écart à la règle ne saurait être toléré de ce comptable, familier de “Littré, Larousse, Dauzat et compagnie» (p. 77), qui tient, parallèlement à son "brouillard", un "cahier de vocabulaire" depuis «trentedeux ans et quatre mois" (p. 33)! Locuteur au langage châtié, Jérôme se révèle également fin connaisseur en matière de style. II sait dès lors estimer à sa juste valeur l'impact du «parler elliptique» d'Athanase (p. 119) ou "apprécie[r] l'obliquité et le lapidaire" d'une pointe (p. 120); il s'oppose à l'interprétation "excessivement littérale" donnée à un trope (p. 121) ou "aux caractères pléonastiques, voire écholaliques inquiétants" d'un "chapelet d'invectives" (p. 113).

Bien qu'admirateur enthousiaste du "télégraphisme» d'Athanase, Jérôme a tendance pour sa part à s'enivrer de mots. Non content de s'exprimer avec clarté et justesse et de recourir au subjonctif imparfait, il lui faut régulièrement, comme à un alcoolique sa ration d'alcool, "sa crise de logorrhée" ( $p, 113$ ), nourrie de "brocards" et "traits verbaux" décochés aux dépens de son prochain. Comme cet «appareil à insulter les gens» (p. 123) «aime bien engueuler [...] quand [il est] en colère, ce qui arrive d'ailleurs assez souvent" ( $p$. 12), les prétextes à verbosité qui feront sa préciosité 's'émoustiller' abondent. Mais, c'est surtout à pratiquer les “raffinements sybaritiques" (p. 68), tels cette description de "la binette" de Passetout (p. 75-76) ou l'interrogatoire au poste de police (p. 142-145), que cette même préciosité se dilate d'aise car alors Chayer, toujours en quête d'assouvissements à sa soif de mots, donne libre cours "à la figure de style dite exténuation" (p. 124) ou, si l'on préfère, à l'amplification. A le voir ainsi s'abandonner sciemment et voluptueusement à la jouissance par les mots, le lecteur découvre qu'il se range, dans le domaine linguistique, parmi "les adeptes de l'exhibitionnisme" (p. 124). A ce penchant d'exhibitionnisme intellectuel s'allie du reste une dispisition au voyeurisme. Celleci se montre manifeste à l'intérêt que suscitent la narration de l'épisode 'érotique' qui s'est déroulé à l'hôtel Mont-Royal entre Madame Bessière 
et Monsieur de Repentigny (p. 49-54) et la relation de la scène sadique qui a eu lieu dans un hôtel anonyme lorsque Athanase a surpris Sylvaine dans les bras d'un galantin (p. 126) ${ }^{39}$. Elle est aussi évidente à la mise en condition précédant les affrontements avec Monsieur de Repentigny ou à l'anticipation de l'engueulade promise entre Passetout et Athanase (p. 86). Exhibitionnisme-voyeurisme: Sylvaine ne conclurait-elle pas que «[c]hacun s'évade comme il peut de la réalité » (p. 21)?

Refusant les "contacts humains", les "amis compréhensifs", "l'affection" (p. 21), Jérôme Chayer leur substitue "les additions, les échecs et le cahier de vocabulaire" (p. 154) auxquels il adjoint, à titre de "soupape numéro quatre et ultime» «le scribouillage» (p. 154). Jusqu'où n'irait pas “un cynique rempli de mauvaise foi" (p. 49) 'pour la forme'! Mais, à se garder en forme au prix d'une demi-mesure humaine et d'une démesure raisonnée, ce joueur de "parties d'échecs mentales" (p. 26), prisonnier de son impuissance physique et de l'univers clos de son intelligence "volontairement raccornie, desséchée» ( $p$. 123), est condamné à se faire échec et mat!

Françoise lqbal Université de la Colombie britannique

1. Le Roman canadien-français, Montréal, Fides, “Archives des lettres canadiennes ", tome III, 1963, p. 339.

2. Gérard Bessette, la Bagarre, Montréal, Le Cercle du livre de France, 1958. Désormais désigné par $B$.

3. Id., le Libraire, Montréal, Le Cercle du livre de France, 1966, p. 142. Désormais désigné par $L$.

4. Id., les Pédagogues, Montréal, Le Cercle du livre de France, 1961. Désormais désigné par $P$.

5. Id., Illncubation, Montréal, Librairie Déom, 1965, p. 134. Désormais désigné parl.

6. I, p. 143.

7. Gérard Bessette, le Cycle, Montréal, Éditions du Jour, 1971, p. 82-83. Désormais désigné par $C$.

8. Il semble évident que nous puissions assimiler, dans la trinité Augustin/Jules/ Weston, l'Américain Weston à Jules. Le lecteur n'a pour s'en convaincre qu'à se rappeler leurs multiples affinités.

9. II est d'abord fait mention, dans le cadre d'un exposé philologique, de l'état de "facticité existentielle" des Canadiens français résultant de leur "mauvaise conscience" à bien parler comme à mal parler la langue française (p. 56). II est ensuite fait mention de "nausée" dans une de ces " tirades " où Augustin, se laissant "prendre à ses propres récits", raconte la perte de sa "virginité" grâce à l'inconscience et à la passivité d'une "créature" croisée au hasard de ses errances dans un "antre existentiel " (p. 64-65).

10. Nous constituons Lebeuf prototype du Canadien français mais nous tenons à préciser que ce Lebeuf s'entend de la superposition des individus qui gravitent autour de lui: Margot, la compagne-serveuse; Bouboule, Bill et compagnie, les balayeurs-compagnons de travail; Gisèle, l'ambitieuse adolescente; les père et mère d'Augustin, bourgeois aisés; la vieille fille Thérèse et sa mère couveuse. Cet échantillonnage est assez représentatif des composantes du Canadien français de l'époque.

11. Cette réalité hostile et opprimante s'exprime avec éloquence dans les physiques presque toujours répugnants des personnages.

12. $B$, p. $56-57$. 
13. La version sillerienne d' "Un Canadien errant ", accompagnée d'exégèse, est un bel exemple d'amplification. Voir $B$, p. 15-17.

14. Se référer aux nombreux "messieurs, jeune homme, docte Lebeuf, mon cher..." des tirades d'Augustin.

15. C. Perelman et L. Oibrechts-Tyteca, Traité de l'argumentation, La nouvelle rhétorique, $2^{\mathrm{e}}$ édition, Bruxelles, Université libre de Bruxelles, Éditions' de I'Institut de socic!ogie, 1970, p. 236-237.

16. Ibid., p. 239-240.

17. Jules avait au préalable eu l'intuition de la verbosité du dilettante: "Lebeuf l'observait d'un air pensif. 'Si seulement il voulait s'appliquer à des choses sérieuses!' Sillery maniait sa langue avec plus d'aisance que lui [...] 'C'est peut-être parce que ses paroles n'ont pas de contenu, précisément'. " (p. 64)

18. Sexe et verbe vont symboliquement de pair chez Augustin dans les deux premiers chapitres: Langevin à ses côtés, il pratique oralement "le dérèglement rimbaldien".

19. Walter F. Otto, Dionysos, le mythe et le culte, traduit de l'allemand par Patrick Lévy, Paris, Mercure de France, 1969, p. 104.

20. Ibid., p. 102-103.

21. Ibid., p. 185.

22. Ibid., p. 149.

23. Penser en fonction de cette antinomie les couples Lebeuf/Margot, Lebeuf/ Gisèle, Weston/Thérèse, Monsieur Sillery/Madame Sillery, Augustin/Langevin, Augustin/Gaston.

24. Signalons au passage que l'oisiveté est un idéal de précieux.

25. Les réflexions et l'action alors consignées sont d'une grande éloquence: "Pour la première fois depuis des mois, à moins que ce ne fû́t des années, j'éprouvais la naive impression que je pouvais encore servir à quelque chose $[. .$.$] (s)i bien$ que, en entrant Chez Trefflé ce soir-là [...] j'inclinai la tête en direction de mon voisin qu'on appelle le père Manseau, un régulier comme moi que je voyais tous les jours depuis un mois sans jamais lui adresser la parole." (p. 55).

26. "L'humour ou la dernière des tristesses", Études françaises, 5, mai 1969, p. 155156.

27. D. Noguez, "l'Humour ou la dernière des tristesses", p. 156.

28. Voir article de Jacques Allard: "/e Libraire de Gérard Bessette ou "comment la parole vient au pays du silence", Voix et images du pays I, Montréal, PUQ, 1970 , p. $51-62$.

29. Voir $P$, p. 123-126.

30. Voir aussi $C$, p. 96 et 98 .

31. Ce nom incite à établir le parallélisme des rôles entre la compagne de naguère et la compagne actuelle, Sophie.

32. Se référer à l'introduction pour le relevé et l'illustration de ces constantes.

33. Voir C, p. 131-133.

34. "Écriture et révolution" dans Théorie d'ensemble, Paris, Seuil, "Tel Quel", 1968, p. 75.

35. Il disait dans le cadre de remarques sur Augustin Sillery: « j'avais remarqué que quand quelqu'un parlait bien, les gens disaient: 'maudit fifi!' [...] Ca donnait à la langue une espèce de signification sexuelle... l'option linguistique..." Le Libraire, présenté et annoté par Jacques Allard, Montréal, Éditions du Renouveau pédagogique, "Lecture Québec".1970, p. 17.

36. Cet appendice est ajouté après la rédaction de notre article dans le but d'intégrer sommairement le dernier roman de Gérard Bessette, la Commensale (Montréal, AS Quinze, 1975). Rappelons que cette cuvre, même si elle vient d'être publiée, fut conçue au début des années soixante.

37. Signalons au passage que le parallélisme des personnages dans les deux cuvres ne se limite pas aux heros. Des rapports s'esquissent avec évidence entre Monsieur Chicoine/h.ionsieur de Repentigny, M. le Curé/M. le vicaire Passetout, Madame Bouthiller/Madame Bessière, le père Manseau/Athanase Bessière.

38. Voir roman p. 34, 110, 113, 143-144, 146, 155, 156.

39. Le cauchemar que Jérôme consigne à la suite de ce récit dévoile, autant par son contenu que par son écriture télégraphique, laquelle infère un dédoublement avec Athanase, l'origine psychologique de l'alliage voyeurisme-exhibitionnisme intellectuel. Celui-ci est "soupape "au refoulement d'une sexualité causé par l'atmosphère affective infantile ( $p .127-129)$. 